\title{
El acompañamiento social personalizado como fórmula innovadora de respuesta a personas con trastorno mental y en exclusión social
}

\author{
Borja Aguirre \\ Asociación Zubietxe \\ <zubietxe@zubietxe.org>
}

Elkarrekikotasuna iradokitzen du laguntzeak gizarte-eskuartzeen alorrean, hau da, laguntzen zaion pertsonak ez du rol pasibo bat hartzen, eta ulertzen da beronen ibilbidea zein den azaltzeko gaitasun propio bat baduela. Eskuartzeetan berrikuntzak gertatzeko gizarte-erakundeek gero eta gehiagoan estimatzen dute kontzeptu hori. Joera bat nabarmentzen da aintzat hartutako pertsonak protagonismo-rol handiagoa izan eta gizarte-ekimenaren erdi-erdian kokatzeko. Zubietxe Elkarteak urteak daramatza proiektu pilotu batean lan egiten gizarte-bazterketa egoeran dauden pertsonei pertsonalizatutako laguntze soziala, eta gure ustez, kontzeptu hori aplikatzeko zailtasunen aurrean soluzio interesgarria eta berritzailea ematen du proiektu horrek. Artikulu hau 2013an egindako proiektuaren sistematizazio-lan baten emaitza izan da.

\section{HITZ-GAKOAK:}

Laguntzea, bazterketa, eskuartzea, sistematizazioa, buru-gaixotasuna.
El acompañamiento, aplicado a la intervención social, es un concepto que sugiere una reciprocidad: la persona acompañada no juega un papel pasivo, sino que se le presupone una cierta capacidad de decidir su propio camino. Se trata de un concepto cada vez más apreciado en las entidades sociales que buscan innovar en sus estilos de intervención. Se observa una tendencia a que la persona atendida cobre un mayor protagonismo y se convierta en el centro de la acción social; a que los recursos se adapten a ella, en lugar de a la inversa. La Asociación Zubietxe lleva varios años trabajando en un proyecto piloto para el acompañamiento social personalizado con personas en exclusión social, un proyecto que consideramos que representa una solución interesante e innovadora ante las dificultades de aplicar ese concepto. El presente artículo es resultado de un trabajo de sistematización de dicho proyecto, realizada durante 2013.

\section{Palabras Clave:}

Acompañamiento, exclusión, intervención, sistematización, enfermedad mental. 


\section{El acompañamiento social personalizado como fórmula innovadora de respuesta a personas con trastorno mental y en exclusión social}

La palabra acompañamiento comienza a escucharse cada vez con más frecuencia en diferentes ámbitos de los servicios sociales; en unas ocasiones, relacionada con nuevas formas y estilos de intervención social; en otras, empujada por tendencias que, desde el entorno de la diversidad funcional, buscan en el acompañamiento una forma de mejorar la calidad de vida y la autonomía de las personas. En el mundo de la exclusión social, sin embargo, no hay muchas referencias al acompañamiento como estrategia central de intervención. Y aún menos pueden encontrarse experiencias concretas de acompañamiento social que eleven la vista sobre su propio trabajo para hacer una reflexión sobre la pertinencia del uso del acompañamiento en estas realidades sociales concretas. Ésta es precisamente la propuesta que late detrás de este artículo.

\section{El Servicio de Acompañamiento Social Personalizado en Zubietxe}

Generalmente los recursos sociales forman parte de una etapa concreta de la intervención psicosocial. Por eso, las intervenciones se dividen u organizan en etapas de menor a mayor estructuración de hábitos, rutinas, tareas, formación -es decir, de menor a mayor autonomía-, fomentando el aprendizaje de habilidades sociales que faciliten la recuperación de las relaciones sociales, familiares o de amigos, y que permitan la inserción laboral y social.

En la Asociación Zubietxe ${ }^{1}$ comenzamos a plantearnos una intervención que no se basase en estos parámetros, sino en la flexibilidad, en la ausencia de etapas prefijadas, en el diálogo educativo y en la asignación de figuras de referencia estables y cercanas. En 2009, comenzamos a testar el ofrecimiento de un sistema de tutorías a un colectivo, el de personas con enfermedad mental y en grave exclusión, como prevención del sinhogarismo, y lo pusimos a prueba con personas que accedieron a participar en él de manera experimental.

Durante este tiempo, hemos descubierto que efectivamente existe un grupo de personas en situación o riesgo de exclusión que necesita un servicio de acompañamiento para estabilizar su situación y mejorar su calidad de vida, un servicio que les ofrezca una persona de referencia en la que apoyarse en los diversos aspectos vitales, atendiendo aquellas demandas que consideran importantes, sin los límites de horario de un centro de día y respetando sus aspiraciones de autonomía personal. El proyecto busca mantener un trabajo socioeducativo de acompañamiento y apoyo

${ }^{1}\langle$ http://www.zubietxe.org〉. individualizado, orientado a satisfacer las necesidades de apoyo social de personas que presentan enfermedad mental junto con otros rasgos de exclusión, vinculándolas con las redes social y sanitaria, y que tenga como horizonte su calidad de vida.

Las y los participantes en él se benefician de una atención personalizada a través de tutorías que se ajustan a sus necesidades individuales en lo que respecta a periodicidad, localización o aspectos abordados. Las destinatarias del Servicio de Acompañamiento Social Personalizado son personas mayores de 18 años, en situación o riesgo de exclusión social, que presentan enfermedad mental grave, con o sin tratamiento; en ocasiones, se incluyen también a algunos de sus familiares en el acompañamiento.

\section{Reflexión interna sobre el proyecto piloto}

En 2012, desde la asociación nos planteamos detenernos un momento para analizar con más detalle los logros de este proyecto piloto, y sus posibles claves de funcionamiento. Junto con la Fundación EDE, que tiene gran experiencia en asesorar en este tipo de reflexiones internas, presentamos a la Fundación Etorbizi un proyecto para investigar durante un año sobre el Programa de Acompañamiento Social Personalizado. La Fundación Etorbizi lo aprobó al considerar que encajaba bien dentro de las propuestas de acción en el ámbito sociosanitario (Gobierno Vasco et al., 2010), y el resultado de ese trabajo es un amplio documento del que resumimos en este artículo sus conclusiones principales. Con ese estudio, hemos tratado de sistematizar la experiencia del tiempo que lleva funcionando el programa, y recoger datos de carácter cualitativo y cuantitativo que nos permitan identificar aquellos elementos clave que inciden en el éxito del servicio, así como valorar la efectividad de este tipo de intervenciones, haciendo especial hincapié en el impacto económico positivo que tienen frente a otros recursos y modelos de intervención.

\section{Definición de acompañamiento social personalizado}

La palabra ‘acompañamiento' es polisémica; también en el mundo de los servicios sociales. En este artículo, nos vamos a referir a una definición concreta de esta palabra: hablamos de un acompañamiento que, aunque tiene un claro horizonte de autonomía y liberación de la persona, no parte de un esquema predeterminado, con etapas definidas, sino que se adecúa al momento presente y a las necesidades cambiantes de las diversas circunstancias vitales. En ese sentido, podemos hablar de una 'ausencia de proceso'.

Se trata de un acompañamiento donde no existen tampoco unos perfiles definidos de personas a las que atender, un acompañamiento que cruza sin pasaporte la frontera entre lo social y lo sanitario, 
entre los diferentes diagnósticos médicos o sociales, entre diversas edades, sexos y orígenes geográficos. No hay compartimentos estancos, ni tampoco una predisposición previa a atender preferentemente unas características de la persona u otras.

Para definir con mayor precisión el tipo de acompañamiento del que estamos hablando, hemos realizado algunas comparaciones con otras tradiciones y estructuras de servicios que existen en nuestro entorno; experiencias tan válidas o más que la nuestra, pero con matices que consideramos necesario señalar.

Existen, en primer lugar, multitud de ejemplos de entidades del sector social, públicas o privadas, que utilizan la palabra acompañamiento para definir un determinado estilo de intervención social. Pueden encontrarse ejemplos en la Fundación Sartu², que lleva desde 2003 hablando de procesos de acompañamiento social y laboral, o de la asociación de empresas de inserción Gizatea³, que escribió en 2008 el documento La mejora de los procesos de acompañamiento en las empresas de inserción (Gallastegi y Martínez, 2011).

El acompañamiento del que estamos hablando en este artículo parte de una base común con estos análisis, ya que lo que se quiere expresar con el concepto de ‘acompañamiento', en términos generales, es una cierta reciprocidad entre la figura del acompañante y del acompañado, elemento este último que, bajo otros entornos conceptuales, como el de 'relación educativa', es una figura mucho más estática y pasiva. A la persona acompañada, en cambio, se le presupone una capacidad de realizar su propio camino.

Compartimos estos planteamientos, nos parece que señalan en la dirección adecuada, y nos sentimos en deuda con las entidades que comenzaron hace años a abrir el camino hacia estas nuevas formas de intervención social. Sin embargo, pensamos que el servicio de acompañamiento que prestamos en Zubietxe, y que aquí estamos analizando, tiene una identidad propia, ya que acompaña a la persona (excluida social) sea cual sea su situación. No nos limitamos a un determinado ámbito, como puede ser el de la inclusión laboral o una determinada asistencia personalizada, sino que acompañamos a la persona en los momentos en que lo necesite, y especialmente en los que más lo necesite. Es obvio que estos momentos suelen coincidir con los de mayores crisis personales, y muchas veces son los más difíciles de acompañar externamente.

Por eso, consideramos que se trata de un acompañamiento integral, no en el sentido de que atiende a todas las necesidades de la persona, al estilo de una institución total, sino que está ahí, acompañando, en las crisis vitales que afectan al conjunto de la per-

\footnotetext{
${ }^{2}\langle$ http://www.sartu.org〉.
}

${ }^{3}$ 〈http://www.gizatea.net〉. sona. Es de esta forma como este acompañamiento consigue ganarse un puesto como referente personal. Los buenos amigos se descubren en los malos momentos, dice la sabiduría popular, y ése es el horizonte simbólico al que queremos aproximarnos.

El acompañamiento que aquí describimos no es tampoco un servicio de intervención en calle, ya que normalmente no se trabaja con gente cuyo contexto es la propia calle, sino con las más diversas entidades, instituciones o espacios sociales. La intervención en calle es un referente histórico en la intuición, hoy muy extendida, de la necesidad que tienen los servicios sociales de salir de las propias estructuras para acudir al contexto social donde se desarrolla la vida y ponerse a la escucha de la persona (Fernández Solís y Castillo Sanz, 2010). Salir del 'despacho' nos proporciona un tipo de relación diferente con la persona, pero además el propio entorno debe conocerse bien y convertirse también en objeto de la intervención social (Brofenbrenner, 2002). La intervención de proximidad es un avance francamente valioso en el mundo de los servicios sociales. Pero con nuestro servicio, a diferencia de este tipo de intervención, buscamos una mayor apertura a otras circunstancias que no sean solamente las del sinhogarismo.

Nuestro modelo tampoco es una prolongación de un recurso sanitario de salud mental. Existen en Bizkaia (Garay et al., 2012) equipos multidisciplinares que incluyen figuras profesionales sanitarias y de salud mental, que disponen de una movilidad que facilita un cierto acompañamiento flexible, basados en las prácticas del tratamiento asertivo comunitario (Martínez Jambrina y Peñuelas, 2007). La aparición reciente de estos equipos es una muy buena noticia, y esperamos que se conviertan en punta de lanza de una psiquiatría más deslocalizada. Nuestro recurso se diferencia de este tipo de equipos en que tiene una mayor pluralidad de fuentes de derivación y va más allá del diagnóstico psiquiátrico de la persona, sin olvidarlo, pero sin ponerlo en el centro de la intervención. También nos diferencia que, como asociación, pertenecemos al tercer sector; esto nos parece importante y volveremos sobre ello más adelante.

El acompañamiento social que aquí describimos se diferencia también del trabajo que realizan las entidades públicas o privadas de atención a personas con enfermedades mentales acompañando a la persona adscrita a su recurso ante sus diversas necesidades concretas. Nuestro servicio de acompañamiento no está unido a un recurso social específico, menos aún si es de alojamiento. Consideramos esto una característica fundamental del servicio. De alguna forma, estamos intentando definir un acompañamiento 'de segundo nivel', donde la persona no es atendida en sus necesidades inmediatas (excepto en situaciones de urgencia), sino que es derivada a otros servicios o recursos varios, sin que eso suponga un traspaso integral de la responsabilidad del caso. La persona sigue manteniendo un seguimiento de intensidad menor, que realiza una función de 'colchón' ante posibles evoluciones o fracasos en 
el proceso. De esta forma, si la persona cambia de domicilio o centro de día, por ejemplo, se le sigue acompañando con la intensidad que necesite, hasta encontrar un lugar que considere propio. Entendemos que este tipo de acompañamiento 'de segundo nivel', a largo plazo, es esencial para asegurar una estabilidad de la persona en sus diversos planos: material, psicológico y social.

Este acompañamiento 'de segundo nivel' tiene puntos en común con el seguimiento generalista que realizan los servicios sociales de base, y no es casualidad que, desde hace años, estos servicios hayan optado por dotarse de equipos de intervención socioeducativa, con profesionales formados en educación de calle, que puedan hacerse cargo de las situaciones complejas de las personas en su propio entorno, y a largo plazo. Con el reciente traspaso a Lanbide de parte de la gestión, se replantea también la necesidad de un acompañamiento más personalizado en todo tipo de situaciones, desde los servicios sociales de base (Duque, 2012). Sin embargo, tal como especificaremos en el apartado referido a la flexibilidad, mantenemos la hipótesis de que el tercer sector está mejor preparado para afrontar situaciones de mucha complejidad, al poder acudir a los más diversos agentes sociales desde una cierta neutralidad institucional, creando tejido sociosanitario desde abajo y sin conflictos estructurales.

No podemos terminar este apartado de definición del acompañamiento sin hacer una referencia a Cáritas Española4, que lleva décadas dedicándose a la intervención social en situaciones de exclusión social. El colectivo de personas que trabaja en estas tareas lleva también años haciéndose preguntas sobre la mejora de su intervención, y contando a menudo con personalidades de alto nivel intelectual para sus reflexiones internas. El resultado es un exhaustivo análisis sobre modelos de intervención; en concreto, hemos podido encontrar documentos de gran profundidad sobre el acompañamiento (merece una especial mención el conocido Marco de intervención con personas en grave situación de exclusión social [Cáritas Española, 2009]) con definiciones claras y matizaciones muy finas sobre el modelo del acompañamiento, con las que sentimos cercanía. Otros documentos avanzan también en este esquema de trabajo (Martínez Rupérez, 2013). Hemos podido comprobar, además, que sus reflexiones no se quedan en aportaciones teóricas, sino que se aplican en proyectos novedosos de acompañamiento (por ejemplo, en el servicio Giltza, en Bilbao) con características realmente interesantes. Valga todo esto como referencia teórica y práctica de lo que queremos expresar.

\section{Valoración cuantitativa mediante la Escala Gencat}

El equipo de trabajo que hemos desarrollado la sistematización y reflexión sobre el modelo de acompañamiento social personalizado hemos entrado en una serie de debates apasionantes sobre las posibilidades y dificultades de realizar una valoración cuantitativa de los progresos de las personas acompañadas. Efectivamente, hemos encontrado muchas dificultades en encontrar un modelo de medición del progreso de una persona en el tiempo, lo que hemos llamado el 'análisis vertical', debido a la radical complejidad de muchas situaciones de exclusión social. De hecho, hemos propuesto una hipótesis sobre las razones profundas de esta dificultad intrínseca. Ahondaremos en esta idea en el último apartado de este artículo.

Nos ha resultado más sencillo y más útil hacer un 'análisis horizontal', es decir, una media de los niveles de bienestar del conjunto de personas acompañadas en un momento dado. Este análisis nos ofrecerá una valoración empírica de la tipología de las personas que atendemos, y de las áreas de su bienestar en las que puede encontrarse mayores carencias, de forma que se conviertan en retos para nuestro trabajo. Éste es el análisis que desarrollamos en el presente apartado.

Entre las muchas opciones que hemos barajado para realizar una valoración cuantitativa del grado de bienestar de las personas atendidas por este servicio, hemos optado por utilizar la escala Gencat (Verdugo et al., 2009), creada por el grupo de investigación interuniversitario en que participan Benito Arias y Laura E. Gómez, del Departamento de Psicología de la Universidad de Valladolid, junto a Miguel Ángel Verdugo, catedrático de la Universidad de Salamanca y director del Instituto Universitario de Integración en la Comunidad (INICO), y Robert L. Schalock, profesor emérito de la Universidad de Nebraska y doctor honoris causa por la Universidad de Salamanca.

La escala Gencat (patrocinada, como su nombre indica, por la Generalitat de Catalunya) persigue evaluar de modo objetivo la calidad de vida de los usuarios de servicios sociales mediante el modelo conceptual propuesto por Schalock y Verdugo (cfr. Verdugo et al., 2009), en el que la calidad de vida se entiende compuesta por ocho dimensiones fundamentales que se relacionan entre sí: bienestar emocional, bienestar físico, bienestar material, desarrollo personal, autodeterminación, inclusión social, relaciones interpersonales y derechos.

La escala está dirigida a los profesionales de los servicios sociales, quienes deben responder a los 69 ítems mediante una escala de frecuencia, basándose en la observación de la persona. Para una correcta valoración de los resultados, hay que escoger entre cuatro baremos que aporta la propia metodología de la escala. Los baremos corresponden a la siguiente tipología de muestras: 
- General.

- Para personas mayores (a partir de 50 años).

- Para personas con discapacidad intelectual.

- Para el resto de colectivos (personas con drogodependencias, $\mathrm{VIH} /$ sida, discapacidad física o con problemas de salud mental).

En nuestro caso, resulta bastante obvio que el baremo más adecuado al perfil de personas que forman parte de la muestra se corresponde al cuarto. Utilizando dicho baremo, hemos realizado escalas Gencat con diez personas usuarias del Servicio de Acompañamiento Social Personalizado. Las personas de la muestra son nueve hombres y una mujer, una proporción enormemente escorada hacia uno de los sexos, que nos da un primer dato sobre el perfil de las personas atendidas y que debe tenerse en cuenta para posteriores conclusiones. Solamente una de ellas es extranjera, al menos ocho tienen enfermedades mentales diagnosticadas, y todas han tenido situaciones problemáticas con algún tipo de droga ilegal o con el alcohol, si bien éste no es el problema principal en todos los casos.

Tras realizar las encuestas y procesar los datos con la metodología Gencat y el baremo escogido, los resultados que hemos obtenido con la muestra son los que aparecen en la Tabla 1. Nótese que todas las cifras representan percentiles, excepto el propio Índice de Calidad de Vida, que es un valor absoluto, y al que acompaña posteriormente su percentil.

La primera conclusión que se puede obtener de estos datos, de forma muy nítida, es que estas personas tienen unos niveles de calidad de vida extremadamente bajos. Estamos hablando de que cinco personas de la muestra (la mitad) se sitúan en el percentil 10, una muestra que además ya había sido baremada como parte de una muestra ajustada a este perfil de personas en exclusión social. El resto de personas, con una excepción, se encuentran bajo el percentil 20. Podemos inferir, por tanto, que trabajamos con un conjunto de personas que están en una situación de precariedad muy alta en muchos aspectos, y que necesitan de algún recurso social para poder mantener un itinerario vital con una cierta dignidad.
Si queremos afinar más en las condiciones de vida de estas personas, podemos desglosar su situación en cada uno de los apartados en que se divide la escala Gencat, y de esta forma, obtener las siguientes conclusiones. En primer lugar, se comprueba claramente que el indicador que muestra unos resultados más bajos es el referido al bienestar material. Son cuatro personas las que se encuentran en el percentil 1 , extremadamente llamativo y que no deja lugar a dudas sobre la precariedad material en que se mueven. Por otra parte, puede observarse que no se trata de un elemento que defina por sí mismo el perfil de las personas atendidas, ya que existe una cierta pluralidad de situaciones, habiendo también una persona que se encuentra en el percentil 63.

Otro indicador con resultados muy bajos es el relativo a la inclusión social. Ocho de las personas estudiadas se encuentran por debajo del percentil 10. Ese indicador hace referencia a aspectos como la relación con el entorno comunitario, familiar, de amistades o la discriminación.

Con estos dos indicadores podemos comenzar a obtener un esquema claro de la situación en que se encuentran las personas atendidas por el Servicio de Acompañamiento Social. Las carencias económicas y el aislamiento social son dos elementos que marcan el recorrido vital con mucha fuerza, retroalimentándose en muchas ocasiones, y apartando a la persona de las posibilidades de una vida normalizada y de las potencialidades de autonomía interna. Efectivamente, estos dos indicadores suponen la 'columna vertebral' de la situación de dependencia y necesidad de apoyo en que se encuentran estas personas.

De cualquier forma, subrayamos la pluralidad de casos; tal como venimos insistiendo a lo largo de toda la investigación, no hay un perfil claro de personas a las que atender, y desde el servicio se procura tener una apertura a situaciones que puedan requerir de apoyo en un determinado momento.

En la dimensión referida a los derechos, encontramos resultados altos, debido a que se han completado los cuestionarios haciendo referencia a los servicios y recursos de apoyo que recibe la persona en el

Tabla 1. Puntuaciones de la muestra en la escala Gencat

\begin{tabular}{|c|c|c|c|c|c|c|c|c|c|c|c|}
\hline & & \multicolumn{10}{|c|}{ Personas usuarias } \\
\hline & & 1 & 2 & 3 & 4 & 5 & 6 & 7 & 8 & 9 & 10 \\
\hline \multirow{8}{*}{$\begin{array}{l}\text { Dimensiones } \\
\text { de la calidad } \\
\text { de vida }\end{array}$} & Bienestar emocional & 9 & 16 & 37 & 16 & 16 & 37 & 25 & 2 & 37 & 16 \\
\hline & Relaciones interpersonales & 9 & 25 & 63 & 37 & 25 & 16 & 16 & 5 & 9 & 9 \\
\hline & Bienestar material & $<1$ & $<1$ & 37 & $<1$ & $<1$ & 5 & 9 & 1 & 16 & 63 \\
\hline & Desarrollo personal & 9 & 16 & 75 & 16 & 5 & 2 & 16 & $<1$ & 16 & 16 \\
\hline & Bienestar físico & 16 & 2 & 9 & 16 & 5 & 9 & 16 & 1 & 37 & 1 \\
\hline & Autodeterminación & 50 & 75 & 50 & 95 & 50 & 84 & 84 & 16 & 50 & 84 \\
\hline & Inclusión social & 16 & 5 & 9 & 37 & 5 & 2 & 2 & $<1$ & 9 & 9 \\
\hline & Derechos & 37 & 63 & 50 & 63 & 50 & 50 & 37 & 9 & 75 & 75 \\
\hline \multicolumn{2}{|c|}{ Índice de calidad de vida } & 74 & 77 & 93 & 87 & 73 & 79 & 82 & 56 & 87 & 86 \\
\hline \multicolumn{2}{|c|}{ Percentil de calidad de vida } & 4 & 6 & 33 & 19 & 4 & 8 & 12 & 1 & 19 & 17 \\
\hline
\end{tabular}

Fuente: Elaboración propia. 
momento de realizarse la encuesta, servicios y recursos que suelen ser de una categoría suficiente como para ver respetados sus derechos y su dignidad. Otra cosa sería si se hubiese tomado como referencia algún momento en el que la persona se hubiese valido por sí misma y no tuviese suficientes apoyos externos; en este caso, probablemente los resultados habrían sido más bajos.

Por último, queremos destacar los altísimos resultados que se obtienen en el apartado de autodeterminación, que hace referencia a la capacidad de elección de estas personas, a sus posibilidades de autoorganizarse o de defender sus propias ideas. Los percentiles indican que todos los casos, menos uno, se encuentran en la mitad superior en cuanto a la capacidad de autodeterminación, y que cuatro casos se sitúan en el $20 \%$ superior. El motivo de estos niveles de autodeterminación se encuentra en que estas personas han sido capaces de mantener su relación con un servicio que les impulsa a tomar decisiones propias y a caminar por su propio pie en muchas de las situaciones en que se encuentran, y a que anteriormente en su vida habrían necesitado de un apoyo. Este resultado quizá también muestra que existe un filtro de las personas con las que se puede realizar un acompañamiento social, un filtro que deja para otros recursos sociales los casos de quienes tienen mayores dificultades para caminar hacia una vida autónoma, por la razón que fuese (desde carencias personales hasta miedos internos).

Nos encontramos, por tanto, que esta escala de Gencat, aplicada a una muestra de las personas que han utilizado el servicio en los últimos dos años, nos ofrece algunas pistas básicas sobre la tipología de situaciones que nos encontramos, y sobre los pasos que se han de dar para lograr una mayor calidad de vida de las personas atendidas.

\section{Claves para el acompañamiento social personalizado}

Durante el trabajo de sistematización y reflexión, hemos realizado un análisis profundo del trabajo realizado en los últimos años. Además del análisis cuantitativo anteriormente descrito, se han realizado análisis de caso, entrevistas a diversos agentes sociales y a grupos de trabajo multidisciplinares. Este esfuerzo nos ha supuesto un año de trabajo, documentado en la monografía que hemos publicado con el apoyo de Etorbizi (Zubietxe y EDE Fundazioa, 2013).

En este artículo no vamos a entrar en detalles descriptivos sobre el proceso realizado, sino que acudiremos directamente a las conclusiones obtenidas, a las claves fundamentales de nuestro Servicio de Acompañamiento. Se trata de los elementos más importantes para una mejora continua de nuestro trabajo, y para una posible implementación de servicios similares en otros contextos. Hay cuatro elementos que consideramos esenciales para un adecuado acompañamiento social personalizado, tal como lo hemos descrito aquí: la información, el vínculo, la flexibilidad y el trabajo en red.

\subsection{La información}

Para realizar cualquier tipo de acompañamiento, es crucial, en primer lugar, conocer bien a las personas a las que se va a acompañar: características y situación personal, diagnóstico de enfermedades, evolución, tratamiento, red sociofamiliar, entorno en el que desarrollan su día a día. También es necesario que el equipo de intervención disponga de los conocimientos básicos sobre las características de los trastornos mentales que aquejan a las personas usuarias, y gocen de apoyo y asesoramiento por parte de los centros de salud mental.

Sin embargo, el acompañamiento en muchas ocasiones se desarrolla bajo un esquema de 'ensayo y error', lo cual quiere decir que nunca sabemos previamente cómo va a encajar la persona en un determinado recurso o cómo va a responder ante una determinada decisión; es solamente a posteriori cuando podremos comprobar si la iniciativa funciona bien o no. En muchas ocasiones aparecen sorpresas, tanto positivas como negativas, por causas desconocidas o imprevisibles. Un ejemplo de ello es cuando una persona usuaria se estabiliza más de lo esperado al entrar en un piso o centro de día, que adopta como 'su lugar', y deja de lado ciertos hábitos negativos. El acompañamiento se diferencia de otras formas de intervención social en que no parte de un diagnóstico de la situación de la persona, sino que sencillamente acompaña a la persona en diferentes contextos, en los que el éxito nunca está asegurado.

Por otro lado, no todos los agentes sociales que se relacionan con la persona usuaria pueden acceder, por diferentes razones, a la información necesaria para una correcta relación de apoyo. Facilitar el flujo de información entre la persona usuaria y el resto de agentes, y entre los propios agentes, es una tarea esencial de la persona que acompaña; crear los canales para que fluya esta información es un trabajo arduo y paciente de creación de tejido social.

\subsection{El vínculo}

Una de las ideas fuerza principales de esta investigación ha sido la del protagonismo del vínculo como herramienta fundamental de trabajo en el acompañamiento. No nos ha resultado fácil definir este concepto; en nuestro estudio, definimos el vínculo basándonos en las ideas de Bowlby (1993) sobre la creación del vínculo en la infancia y las consecuencias de su ausencia. Este concepto, además, debe estar enmarcado en un contexto de tratamiento terapéutico, donde su significado se aproximará al de adherencia. En todo caso, está claro que estamos hablando de un tipo de relación donde la persona acompañada tiene un cierto grado de confianza en el 
acompañante, y esta relación de confianza es capaz de mantenerse en el tiempo, superando incluso situaciones conflictivas.

Esta importancia del vínculo diferencia a este acompañamiento del que se realiza en otros ámbitos, como el de las discapacidades físicas o las personas mayores, por ejemplo. Efectivamente, el ámbito de la exclusión social tiene una característica propia, que es la ruptura de las relaciones sociales y la sensación de intensa soledad y marginación social (entiéndase esta palabra en su sentido original) que sufre la persona excluida, y ante la que adopta diversas estrategias. El vínculo pretende abrir una grieta en ese muro de separación, y atravesarlo con pequeños lazos que esperan multiplicarse y abrir espacios de contacto con otros agentes sociales.

No es sencillo encontrar indicadores sobre el grado de consecución del vínculo, pero quizá una forma de definirlo sea precisamente la disposición de la persona acompañada a volver a la persona acompañante una y otra vez, pese a los diferentes momentos y contextos vitales.

El vínculo educativo debe ser lo más claro posible para ambas partes, es decir, que no genere confusión, en el sentido de que alguna de ellas crea que estamos ante una relación de amistad o similar. Por supuesto, la parte afectiva va a aparecer en muchas ocasiones y con diferentes formas, pero es función de la persona profesional manejarla adecuadamente sin permitir que condicione la intervención. Hay que tener en cuenta que, con personas con trastornos mentales severos, el vínculo afectivo es en ocasiones difícil y muy débil, por lo que es más fácil que se rompa. Por el contrario, en los casos de 'fragilidad emocional' el peso de lo emocional es muy importante y el vínculo afectivo es mayor.

Unido a lo anterior, es importante partir del respeto al proyecto vital a la persona usuaria. Sabemos que las expectativas de las personas usuarias no siempre están ajustadas a su realidad, lo que les puede provocar frustración, y, por nuestra parte, podemos caer en el error de querer dirigir permanentemente sus actos. Este respeto a la persona se complementa con la necesaria empatía, y se convierte en la columna vertebral de un acompañamiento que quiere poner a la persona en el centro, devolviéndole en el mayor grado posible la responsabilidad de sus decisiones y el timón de su propia vida.

Hemos contemplado con claridad en este estudio que el momento de la acogida es clave para establecer el vínculo. Facilitar la creación de este vínculo en los primeros pasos de la relación es muy importante. En este sentido, es importante realizar la acogida en el propio contexto de la persona usuaria o bien realizar lo que se llama acogida mutua, en un contexto que no es propio de la persona usuaria ni del educador. Hacerlo de esta manera agiliza la creación del vínculo con la persona.
Por esta razón, y porque las personas normalmente están en situación de urgencia cuando llega a ellas el servicio, inicialmente la persona casi siempre se deja llevar sin hacerse muchas preguntas ni mostrar muchos miedos. Se acepta el acompañamiento de una forma casi instantánea, lo cual a veces tiene matices sorprendentes.

Esta capacidad de crear un vínculo con la persona acompañada depende en un alto grado de las capacidades de quien realiza la intervención: conocimientos, experiencia y habilidades son grandes elementos facilitadores de la intervención. Unido a esto, es importante manejar algunas claves para generar el vínculo, tales como crear confianza, sinceridad mutua, libertad, empatía, escucha; y, especialmente, saber esperar el momento y conseguir que quienes están siendo acompañados sean quienes tomen la decisión de acudir a un determinado recurso u otro.

\subsection{La flexibilidad}

La flexibilidad es otro de los elementos que se han considerado claves en el proceso de acompañamiento. Para realizar una intervención que realmente esté centrada en la persona, es preciso ser flexibles en las acciones realizadas, en los horarios y en la intensidad que la intervención requiere, entre otros factores. Esto es especialmente importante en la primera fase de la intervención, en el momento de creación del vínculo, pero puede decirse que la flexibilidad es el único protocolo estable en la relación de acompañamiento.

En el plano relacional, la flexibilidad necesita una característica previa, que es la capacidad de escucha, conocer las necesidades de la persona. Se llega a la flexibilidad con las personas tras escucharlas con los mínimos prejuicios posibles, y tomar posteriormente las decisiones pertinentes. Se parte de la escucha a la persona como centro, y se ve, posteriormente, que la única forma que se puede responder es mediante la flexibilidad.

Al comenzar el proceso generalmente no existe una demanda inicial explícita por parte de las personas usuarias, quienes no poseen expectativas. Y, de alguna forma, tampoco hay proceso; más bien, lo que existe es una ausencia de proceso, en el sentido de que no hay un guión o un itinerario compartido. Cada persona es un mundo y hay que responder a su realidad con mucha flexibilidad y creatividad.

Los itinerarios son en forma de espiral, con retrocesos y avances; y en este modelo de intervención, es clave el acompañamiento. Normalmente, una vez que la persona sale de su situación inicial de urgencia y se estabiliza, suelen aparecer de nuevo factores desencadenantes de nuevas crisis. Esto requiere gran flexibilidad en la intervención.

En el terreno organizativo, podemos llegar a más conclusiones interesantes. Para garantizar la flexibilidad en la intervención, es un requisito previo 
que la organización en la que está inserto el servicio también sea ágil y flexible. Sólo de esta manera será capaz de adaptar los recursos a las necesidades de las personas usuarias. Éste es uno de los factores que nos hace pensar que el tercer sector está más capacitado para realizar acompañamientos flexibles que otros entornos más institucionales o administrativos. Nos encontramos a diario con entidades que tienen dificultades para 'salirse del protocolo' en una determinada intervención, o de crear perfiles de atención más difusos. También nos encontramos continuamente con conflictos institucionales donde diferentes estructuras luchan entre sí por mantener su identidad y su espacio de intervención concreto.

No es el objeto de este estudio analizar por qué el ámbito público tiene una mayor tendencia a la burocratización y a la clasificación en protocolos de toda su actividad; probablemente la sociología de Weber (2013) y otros autores podría aportar luz sobre este punto. Sugerimos solamente la intuición de que la responsabilidad pública empuja hacia una definición cerrada y precisa del propio ámbito de actuación, y que muchos profesionales de la rama pública se quejan de la dificultad de crear espacios multidisciplinares y abiertos. También nos remitimos a la realidad cotidiana, donde el tercer sector (que, por otro lado, tampoco se libra -nos libramos- de una tendencia a la esclerotización) disfruta, en general, de una mayor libertad de movimientos entre diferentes servicios, recursos, oficinas y equipos profesionales.

En nuestro caso, la posibilidad de ajustar la intervención a la intensidad que requiere en cada momento se garantiza por la flexibilidad existente en la organización para adaptarse a las necesidades de las personas. Se ha tratado de superar la visión inicial de 'falta de encaje en el recurso' y pasar a 'adaptar el recurso a las necesidades de las personas'. Es necesario estar constantemente ajustando los servicios y la intervención, y hay que estar reinventando los servicios. Para ello, es necesaria una estructura que lo permita, que no sea muy rígida; la clave es poner en el centro a la persona y que el equipo educativo tome las decisiones a través de lo que escucha, no de la memoria del año anterior.

Todo ello sólo se consigue con un gran esfuerzo por parte de la organización y analizando constantemente la realidad, planteando hipótesis, poniendo en marcha acciones concretas, desarrollando proyectos piloto, evaluando los resultados y consiguiendo su implementación. La organización debe facilitarlo y promover la improvisación constante siempre que sea necesario.

Uno de los aspectos más valorados, tanto por parte de las personas usuarias como por la propia organización, es que el servicio de acompañamiento esté inserto en una entidad que pueda ofrecer servicios de apoyo. Eso permite un mejor 'engrase' de las tareas del acompañamiento y facilita la incorporación de las personas a los recursos. En concreto, el centro de día de Zubietxe es un apoyo muy importante: el propio espacio, las y los educadores, el comedor, los y las profesionales de trabajo social y jurídico.

Como consecuencia del punto anterior, no parece viable que el recurso de acompañamiento sea totalmente autónomo. Parece más recomendable que esté inserto en una entidad más amplia que disponga de otros recursos complementarios. Además, será necesario crear determinados protocolos de trabajo con profesionales externos.

Finalmente, destacamos como un aspecto importante de la flexibilidad el manejo de las excepciones. $A$ veces hay que pedir un favor a algún profesional de nuestra entidad o de la red para que haga una excepción, porque estamos ante un caso complejo y con pocas vías de salida. No todos los y las profesionales saben manejar el arte de la excepción, saber cuándo utilizarla y cuándo no. Y, en gran parte, lo que se valora no es la petición concreta, sino la persona que la hace: de ahí la importancia de un vínculo entre profesionales que vaya algo más allá de lo institucional. Esto nos da pie al siguiente punto.

\subsection{El trabajo en red}

El trabajo en red se convierte en el espacio vital del acompañamiento, un trabajo en red en el que, como hemos visto, la persona acompañante debe saber moverse con agilidad y flexibilidad. Esta flexibilidad y el manejo de las excepciones de las que hablamos en el punto anterior está relacionado con la implicación de todo el colectivo de profesionales y agentes sociales. Parece haber determinados profesionales entre los cuales existe una mayor relación por razones no tanto (o no sólo) afectivas, sino profesionales, por forma y estilo de trabajo, por su disposición en el manejo de las excepciones, por el papel que juegan... Hay profesionales con los que se tiene mayor confianza y hay que contar con ello. Conseguir la implicación de los y las profesionales es clave para trabajar en red.

Por tanto, el trabajo en red no solamente depende de las funciones de la institución, sino de las personas concretas que trabajan en ella. Teóricamente una institución debería cumplir las mismas funciones independientemente del profesional que trabaje en ella, pero la realidad no es así, y hay que tenerlo en cuenta. Esto no significa despreciar la institución y su formalización de procesos, que es válida y necesaria para conseguir una universalización y equiparación de los servicios; simplemente estamos poniendo de relieve una realidad práctica que no siempre es apreciada: la diversidad existente en el interior de las instituciones y la forma de orientar esta pluralidad en beneficio de la persona acompañada.

Por todo ello, es fundamental para el profesional del acompañamiento conocer bien la red: por un lado, las instituciones y recursos; y por otro, las personas.

Otra de las claves que en muchas ocasiones tienden a olvidarse es la de los cambios sistémicos en el 
entorno de la persona. Al ir cambiando la situación de la persona, puede ir cambiando también su contexto: familiares, personas conocidas, recursos de su alrededor se reorganizan para la nueva situación. Por ejemplo, un familiar cercano puede volver a acercarse a la persona acompañada al verla recuperada, $y$ entonces aparecen nuevos problemas y situaciones, nuevos retos y nuevos espacios de relación con otros recursos y servicios sociales.

Si hablamos del contexto sociosanitario en el que se desenvuelve la persona acompañada, es importante señalar que el profesional que acompaña se va a convertir en un referente, no solamente para la persona acompañada, sino también para los diversos recursos que giran en torno a ese profesional. Cuando se necesita contactar con la persona acompañada, se suele llamar al profesional de referencia. Esta labor del acompañante como 'referente' mínimamente estable ante las instituciones y entidades es una forma de generación de tejido social, en concreto, de creación de red sociosanitaria, no como estructura o como teoría, sino como praxis cotidiana. El tejido sociosanitario, así entendido, es una tupida red creada por centenares de relaciones interpersonales e intersectoriales que se han generado una por una, con mimo e interés. Este nivel micro y meso es una realidad de la red sociosanitaria (Ararteko, 2008).

\section{Una consecuencia inesperada de nuestra investigación: el 'efecto Noé'}

No queremos finalizar este artículo sin aportar una de las que consideramos que ha sido las conclusiones más interesantes y novedosas de nuestra investigación, consecuencia de los intensos debates que se han generado dentro de la asociación y en los diversos foros de trabajo. Conclusión que no es específica del tema de este artículo, el acompañamiento social, sino que más bien está relacionada con la exclusión social en general y con las investigaciones que sobre ella podamos realizar. Se trata de la hipótesis de que los contextos de exclusión social son especialmente refractarios a mediciones y análisis cuantitativos 0 estadísticos.

Efectivamente, a la hora de plantear la metodología de nuestra investigación, y en concreto al debatir sobre las herramientas más adecuadas para medir el éxito o fracaso de la intervención (medido como el progreso personal en los itinerarios de inserción a través de instrumentos como las baterías de indicadores, por ejemplo), encontramos que no nos satisfacía ninguna de las posibles propuestas. Barajamos diferentes tipos de escalas de medición, considerando algunas más válidas que otras, pero todas nos provocaron una cierta insatisfacción al comprobar que muchos de los resultados objetivados por las muestras no mostraban una correspondencia con nuestras impresiones subjetivas sobre el estado de la persona evaluada, y menos aún sobre las evoluciones positivas o negativas que mostraba la persona en su trayectoria vital.
Nos movía la intuición de que las personas en exclusión social son un colectivo que mantiene algunas diferencias profundas con el resto de extractos sociales, diferencias que le proporcionan un estatus único y peculiar. De alguna forma, las vidas de las personas que se encuentran en esta situación sufren unos vaivenes en el tiempo que están fuera de lo común; sus vidas son extremadamente azarosas y caóticas. A menudo no existen 'referencias' sobre lo que su vida fue o podría llegar a ser, y viven en un entorno de inseguridad radical, donde cualquier pequeño cambio en su contexto personal podría tener consecuencias enormes y profundas. Esto provoca que los itinerarios vitales de estas personas sufran de altibajos desmesurados, y que la medición empírica sobre sus posibles progresos personales sea prácticamente imposible de encontrar.

Buscando una herramienta científica a la altura de estas dificultades, nos encontramos con la propuesta del matemático y estadístico Benoît Mandelbrot ${ }^{5}$. Mandelbrot (1996) distingue entre dos tipos de azar: el benigno y el salvaje. En los azares benignos, lo que es impredecible a escala local o micro se hace determinado y predecible a escala global o macro. Por ejemplo, no podemos saber a priori la altura de una persona escogida al azar, pero si escogemos varios cientos de personas, la media es bastante consistente y predecible. En los azares demasiado erráticos, en cambio, el paso a los grandes números ya no es capaz de proporcionar las bases del control y la predicción. Por ejemplo, no tiene sentido calcular la media del número de habitantes de los municipios de un país, donde una sola ciudad (pongamos Buenos Aires) puede representar la cuarta parte del conjunto total. El hecho de incluir o no a un sólo municipio (Buenos Aires) en la muestra cambia totalmente el resultado (la media).

Mandelbrot observaba que, como en el Diluvio Universal padecido por Noé, ciertos acontecimientos (en la economía financiera y en otros ámbitos sociales) tienen una magnitud mucho mayor que el resto; son elementos que 'se salen de la tabla' o hechos que ocurren de golpe y barren todo a su paso. Por eso, Mandelbrot bautizó a este tipo de situaciones como el 'efecto Noé'. Un alumno de Mandelbrot, el matemático e inversor financiero Nassim Taleb, ha vuelto a atraer en los últimos años el interés científico y social sobre estos conceptos, que él ha rebautizado como 'cisnes negros' (Taleb, 2008). En un sentido estadístico estricto, en este tipo de modelos los sucesos más improbables son siempre los más importantes.

5 Benoît Mandelbrot es conocido por ser uno de los fundadores de las llamadas matemáticas del caos. Su artículo "La variación de ciertos precios especulativos”, sobre el precio del algodón” (1963), se considera, junto con el texto del meteorólogo Edward Lorenz "¿Puede el aleteo de una mariposa en Brasil provocar un tornado en Texas?" (1972), el inicio de un campo de estudio que ha cambiado la forma de ver las matemáticas (quizá ya no sean 'ciencias exactas') y posiblemente la ciencia en general. Mandelbrot es famoso también por haber inventado el término 'fractal' y por la sorprendente belleza de las funciones matemáticas llamadas conjuntos de Mandelbrot, que, con sus complejos dibujos llenos de colorido, han mostrado la belleza de los sistemas caóticos y su parecido con la propia vida. 
No hay una 'media' o un 'atractor' que utilizar como referencia, y no es sencillo -en ocasiones es imposible- encontrar un dato o un conjunto de datos que definan el sistema.

Nuestra hipótesis de trabajo es que la vida de las personas en situación de exclusión, la anomía de sus relaciones sociales, la inseguridad crónica en la que se desenvuelven, la falta de referencias sociales a las que unirse, genera un contexto caótico que encaja bien en la descripción de los sistemas complejos sujetos al azar salvaje que describe Mandelbrot. La intuición y la experiencia cotidiana nos indican que, efectivamente, las personas que viven en estos contextos están mucho más expuestas a acontecimientos inesperados de gran impacto, que pasan por su vida con el efecto de un tsunami o un diluvio; y que son mucho más vulnerables a los efectos de pequeñas variaciones o errores en su entorno.

Si esto fuera así, nos encontraríamos con una realidad social que muestra una gran dificultad en ser analizada empíricamente; aún más, la exclusión social sería un ámbito de análisis en el que ni siquiera podríamos tener seguridad de encontrar una relación causa-efecto de sus experiencias vitales. Lo que puede mostrarse de forma empírica es precisamente los límites concretos de cualquier análisis cuantitativo del progreso personal.

No nos corresponde avanzar sobre esta hipótesis, utilizando los métodos científicos adecuados para demostrar o negar su validez; ésa sería más bien una tarea apropiada para un estudio dentro del ámbito universitario. Nuestro único objetivo ha sido dejar constancia de una dificultad real y de una posible propuesta de adecuación teórica a una realidad empírica.

En la práctica, de confirmarse la hipótesis, algunas consecuencias de este modelo estadístico nos habrían resultado de interés para nuestro trabajo cotidiano. Pese a tratarse de una supuesta limitación en nuestra capacidad de conocer la realidad, el efecto Noé acarrea varias conclusiones interesantes. En primer lugar, el trabajo con las personas en situación de exclusión social debe hacerse eliminando los juicios de valor y poniendo entre paréntesis las narrativas creadas sobre las circunstancias de la persona; tanto las narrativas creadas por la propia persona como aquellas generadas por su entorno o sociedad. No puede darse por sentado ningún escenario básico, normalizado, que sirva de punto de vista privilegiado desde el que analizar la situación de estas personas. Incluso el sistema de normas morales, aparente- mente estable, se relativiza al romperse la relación causa-efecto y desdibujarse la capacidad de unir determinadas acciones con determinadas consecuencias. El pasado puede relativizarse.

En segundo lugar, hay que trabajar sin obsesionarse por los resultados. Mucho menos por obtener una alta calificación en un conjunto de indicadores de progreso, creados por agentes externos o subvencionadores, que pretenden cuantificar los logros concretos obtenidos. Los indicadores de progreso son útiles y necesarios para una mejora de la calidad del trabajo, pero no deben confundirse con la propia metodología, que tiene su propia lógica, más relacionada con los pequeños pasos y el día a día. El presente lo es todo.

En tercer lugar, las personas en contextos de exclusión muestran una enorme vulnerabilidad a hechos inesperados en sus vidas. La tarea fundamental del acompañamiento personalizado podría ser la de aportar un cierto tipo de seguridad que incluya la capacidad de gestionar estos hechos inesperados y de gran impacto. No se trata solamente de ayudar a gestionarlos, sino especialmente de prevenirlos, desarrollando un cierto instinto ante posibles circunstancias negativas que puedan aparecer en la vida de la persona. De la misma forma que se contratan seguros de incendios al comprar una casa, el acompañante debería crear mecanismos de prevención que aminoren los efectos de cambios inesperados en el entorno. El alumno de Mandelbrot, Taleb, se hizo millonario con la crisis de 2008, en la que todo el mundo perdió dinero, gracias a su adecuada y novedosa forma de gestión del riesgo. La persona o equipo acompañante pueden convertirse en una figura de apoyo a la que recurrir en los momentos de mayor quiebra personal. El futuro puede ser menos arriesgado.

\section{Epílogo}

Soplan vientos de cambio en la intervención social. Una nueva revolución parece asomar en el horizonte: la revolución de la centralidad de la persona, de la confianza en que la persona acompañada va a poder recuperar su autonomía, su capacidad de asumir el timón de su propia vida. En la asociación Zubietxe, hemos querido poner nuestro granito de arena en esta ola, desarrollando un pequeño proyecto y dedicando un tiempo a la reflexión sobre el trabajo hecho. Esperamos que en el futuro seamos muchos los proyectos que caminemos abriendo paso en esta dirección, y que vayamos encontrando en esta aventura nuevos acompañantes. 
ARARTEKO (2008): Informe extraordinario sobre atención sociosanitaria: una aproximación al marco conceptual y a los avances internacionales y autonómicos, Vitoria-Gasteiz, Ararteko.

BOWLBY, J. (1993): El apego, Barcelona, Paidós Ibérica.

BROFENBRENNER, U. (2002): La ecología del desarrollo humano, Barcelona, Paidós.

CÁRITAS ESPAÑOLA (2009): Marco de intervención con personas en grave situación de exclusión social, Madrid, Cáritas Española.

DUQUE, J. M. (2012): “Redescubrimiento de los servicios sociales de atención primaria: hacia un (nuevo) modelo de atención personal y comunitaria", Zerbitzuan, $n^{\circ}$ 52, págs. $23-44$ [<http://dx.doi. org/10.5569/1134-7147.52.02〉].

FERNÁNDEZ SOLÍS, J. D.; y CASTILLO SANZ, A. G. (2010): La educación de calle. Trabajo socioeducativo en medio abierto, Bilbao, Desclée de Brouwer.

GALLASTEGI, A.; y MARTÍNEZ, N. (coords.) (2011): La mejora de los procesos de acompañamiento en las empresas de inserción, Gizatea, Bilbao [<http:// www.gizatea.net/doc_up/gizatea/Procesos. $p d f>]$.

GARAY, M. et al. (2012): "Evolución del Programa de Asistencia Psiquiátrica a Personas Sin Hogar con Enfermedad Mental Grave en el municipio de Bilbao", Zerbitzuan, $n^{\circ}$ 52, págs. 179-190 [khttp://dx.doi.org/10.5569/1134-7147.52.12〉].

GOBIERNO VASCO et al. (2010): Documento marco para la elaboración de las directrices de la atención sociosanitaria en la Comunidad Autónoma Vasca. Diciembre 2010 [rhttp:// www.osakidetza.euskadi.net/contenidos/ informacion/atencion_sociosanitaria/ es_atencion/adjuntos/Atencion $\% 20$ Sociosanitaria\%20-castellano- $\% 20$ LOGOS-28-2-11.pdf>].

LORENZ, E. N. (1972) "Predictability: Does the flap of a butterfly's wings in Brazil set off a tornado in
Texas?”, [comunicación en congreso], American Association for the Advancement of Science, $139^{\text {th }}$ Meeting [<http://eaps4.mit.edu/research/ Lorenz/Butterfly_1972.pdf〉].

MANDELBROT, B. (1996):“Del azar benigno al azar salvaje”, Investigación y Ciencia, no -246, págs. 14-21.

- (1963): "The variation of certain speculative prices", The Journal of Business, vol. $36, \mathrm{n}-4$, pags. 394-419.

MARTÍNEZ JAMBRINA, J. J.; y PEÑUELAS CARNICERO, E. (2007): "Tratamiento asertivo comunitario: el modelo Avilés", Archivos de Psiquiatría, $n^{\circ}$ 70, págs. $77-82$ [<http://www. archivosdepsiquiatria.es/index.php?journal=a dp\&page $=$ article $\& o p=v i e w \&$ path []$=21\rangle]$.

MARTÍNEZ RUPÉREZ, M. (coord.) (2013): El arte de acompañar/nos. Procesos y metodología, serie: Cuadernos de Formación, Madrid, Cáritas Española [<http://www.caritasburgos.es/ biblioteca/docusnoticias/elarte.pdf〉].

TALEB, N. N. (2008): El cisne negro: el impacto de lo altamente improbable, Barcelona, Paidós Ibérica.

VERDUGO ALONSO, M. A. (dir.) (2009): Escala GENCAT. Formulario de aplicación de la Escala GENCAT de Calidad de Vida, Barcelona, Generalitat de Catalunya, Institut Català d'Assistència i Serveis Socials [rhttp://inico.usal.es/ documentos/EscalaGencatManualCAST.pdf〉].

WEBER, M. (2013): La ética protestante y el espíritu del capitalismo, Madrid, Akal.

ZUBIETXE; y EDE FUNDAZIOA (2013): Proyecto piloto para el acompañamiento social personalizado como formula innovadora de respuesta a personas con trastorno mental y en exclusión social, Zubietxe [<http://zubietxe.org/wp-content/ uploads/2013/12/Documento-Zubietxe.doc〉]. 